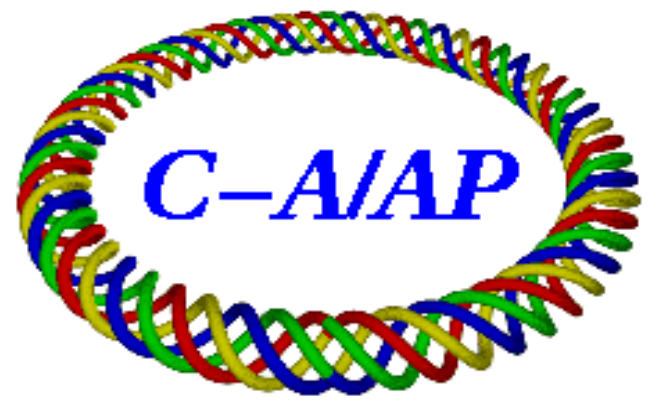

Collider-Accelerator Department Brookhaven National Laboratory

Upton, NY 11973 


\section{Plasma Micro-Stability Analysis for the RHIC Electron Beam Cooler}

\section{Ady Hershcovitch}

Plasma kinetic theory is applied to the RHIC electron beam cooler to examine the potential for plasma microinstabilities. Two sources of "free energy" are identified: density and temperature gradients. It is shown that the RHIC electron beam cooler is stable (based on present parameters) against any plasma microinstabilities that can be driven by either of these sources during an interaction period.

\section{Introduction}

Before stability analysis is performed on any plasma (i.e., "plunging" into calculations), sources of free energy must be identified. If there are sources of free energy, potential for instabilities exists. In the bunch rest frame of the RHIC electron beam cooler, there are density gradients in a radial direction with a longitudinal magnetic field, which may drive drift type modes; longitudinal forces that may affect the velocity distribution function; and, anisotropic velocity distribution that has the potential to drive instabilities. Plasma kinetic theory is used to evaluate the potential for instabilities that may be driven by these sources of free energy.

Unlike atomic physics where the Schrödinger equation can be used to define and become the basis for solving a problem, plasma physics does not have a master equation. Instead, a set of equations must be chosen based on the best guess as to which equations are most relevant. There are two basic levels of theoretical description: (1) Microscopic description, also referred to as plasma kinetic theory, which is based on phase space distribution of plasma particles. One of the main virtues of plasma kinetic theory is the natural manner in which finite-temperature effects are included. (2) Macroscopic or fluid description, which is based on moments of the Liouville or the Boltzmann equations, some of Maxwell's equations and Ohm's law. Although microscopic quantities are difficult to measure, they frequently play a dominant role in determining macroscopic properties. The choice of equations in either theory is based on the problem to be solved.

By its definition, plasma is a collection of particles whose statistical properties are determined by multiple Coulomb interactions. Mathematically, it can be expressed as $n \lambda_{D}^{3} \gg>1$, where $n$ is particle density and $\lambda_{D}$ is the Debye length. Basically, there should be many particles in a Debye sphere to have plasma collective effects. The possibility of plasma collective effects is what distinguishes plasmas from other collections of charged particles. In the RHIC E-Cooler expected particle rest frame (PRF) values ${ }^{1}$ are $n \lambda_{D}^{3} \approx 1.2 \times 10^{5}$ for ions and $n \lambda_{D}^{3} \approx 6 \times 10^{7}$ for electrons.

In addition to meeting the general requirement for plasma collective effects, the Debye length must be smaller than a physical system for plasma kinetic theory analysis to be valid. In the PRF of the RHIC E-Cooler bunch lengths for ions and electrons are 30 meters and 3 meters respectively. Bunch radius for both ions and electrons is $2 \mathrm{~mm}$. $\lambda_{D}$ is $^{1} 3.477 \mathrm{~mm}$ for ions and $2.381 \mathrm{~mm}$ for electrons. Thus, $\lambda_{D}$ is smaller than the bunch 
diameter. Finally, attention must be paid to ensure that analyzed phenomena occur within appropriate time scales. Interaction time $\mathrm{J}$ in PRF frame is $10^{-9} \mathrm{sec}$.

\section{Elements of Plasma Kinetic Theory}

Modern plasma kinetic theory has its foundation in the Vlasov ${ }^{2}$ model of plasma waves. In this model particle discreteness is lost, i.e., the number of particles $\mathrm{N} \rightarrow \infty$, while their charge and mass $\mathrm{q}$ and $\mathrm{m} \rightarrow 0$. But $\mathrm{qN}$ and $\mathrm{mN}$ remain constant. It is as if particles are being chopped into smaller and smaller fragments to reach the so-called "mush" limit. Since the plasma does not have discrete particles in this limit, the plasma is collisionless. However, it is not correlationless. The various components (particles) of the plasma interact with each other via waves, i.e., their electric fields.

Like any many-body system, a chain of statistical equations, which could or could not be closed, can describe a collection of charged particles. However, in plasma (where $n \lambda_{D}^{3}>>1$ ) taken to the mush limit, statistical chains of the Liouville equations can be simplified by neglecting terms of the order of $1 / n \lambda_{D}^{3}$. To this order the kinetic equation for the $\mathrm{j}^{\text {th }}$ species distribution function is

$\left(\frac{\partial}{\partial t}+\vec{v} \bullet \vec{\nabla}+\frac{q_{j}}{m_{j}} \vec{E} \bullet \vec{\nabla}_{\bar{v}}\right) f_{j}(\vec{r}, \vec{v}, t)=0$

Where $f_{j}$ is the distribution function for $j^{\text {th }}$ species. Variables with arrow above them denote vector quantities. Equation 1 is called the Vlasov equation. The interaction among the particles appears through E, the average electric field, which can be computed self consistently through Maxwell's equations. With

$\nabla \bullet \vec{E}=\sum_{j} \frac{q_{j} n_{j}}{\varepsilon} \int f_{j}(\vec{r}, \vec{v}, t) d \vec{v}$

Where $\varepsilon$ is the permittivity. Equations 1 and 2 can, in principle, be used to determine stability due to a small perturbation in $f(\vec{r}, \vec{v}, t)$.

This procedure can be generalized to include externally applied and internally generated magnetic fields.

$$
\begin{aligned}
& \left(\frac{\partial}{\partial t}+\vec{v} \bullet \vec{\nabla}+\frac{q_{j}}{m_{j}}(\vec{E}+\vec{v} \times \vec{B}) \bullet \vec{\nabla}_{\vec{v}}\right) f_{j}(\vec{r}, \vec{v}, t)=0 \\
& \nabla \bullet \vec{E}=\sum_{j} \frac{q_{j} n_{j}}{\varepsilon} \int f_{j}(\vec{r}, \vec{v}, t) d \vec{v}+\frac{\rho_{e x t}}{\varepsilon}
\end{aligned}
$$




$$
\begin{aligned}
& \nabla \times \vec{B}=\frac{\partial \vec{E}}{\partial t}+\sum_{j} \frac{q_{j} n_{j}}{\varepsilon} \int f_{j}(\vec{r}, \vec{v}, t) d \vec{v}+\frac{\vec{J}_{e x t}}{\varepsilon} \\
& \nabla \times \vec{E}=\frac{\partial \vec{B}}{\partial t}
\end{aligned}
$$

Where $\mathrm{J}$ and $\rho$ are current and charge density respectively. B represents magnetic field. Equations 3,4,5, and 6 form a closed set. A major advantage of this theory is the ease with which non-neutral plasma, or even single species plasma can be treated.

Landau $^{3}$ was the first to solve the Vlasov equation. He considered only electrostatic modes in infinite homogenous plasma with no external electric or magnetic fields. Landau examined the effect of a small perturbation on the distribution function $\widetilde{f}_{j}(\vec{r}, \vec{v}, t)$ that results in a small perturbed potential $\varphi$ that either damps (stable modes) or grows (unstable modes). Mathematically, equations 1 and 2 are solved as a linearized initial value problem. Both equations are linearized with

$$
f_{j}(\vec{r}, \vec{v}, t)=f_{o j}(\vec{v})+\widetilde{f}_{j}(\vec{r}, \vec{v}, t) \quad \text { and } \quad \widetilde{\vec{E}}=-\nabla \varphi
$$

to yield

$$
\frac{\partial}{\partial t} \widetilde{f}_{j}(\vec{r}, \vec{v}, t)+\vec{v} \bullet \nabla \tilde{f}_{j}(\vec{r}, \vec{v}, t)-\frac{q}{m} \nabla \varphi \bullet \nabla_{\vec{v}} f_{0_{j}}(\vec{v})=0
$$

and

$$
\nabla^{2} \varphi=\sum_{j} \frac{q_{j} n_{j}}{\varepsilon} \int f_{j}(\vec{r}, \vec{v}, t) d \vec{v}
$$

$\widetilde{f}(\vec{r}, \vec{v}, t)$ and $\varphi$ are the only unknowns in equations $7 \& 8$. To solve these equations, they are next reduced to algebraic equations by Fourier-Laplace transforms. After solving the algebraic equations for $\varphi$, transform inversion must be carried out (in principle) to determine the system response. There is, a problem with the Laplace inversion, since its integration contour must be above all poles resulting in exclusion of the $\varphi(\vec{k}, \omega)$ singularities. Landau developed the technique for analytical continuation of the contour, which was a major breakthrough. Now time asymptotic response can be found by considering the highest pole, which due to the zero of the denominator of $\varphi(\vec{k}, \omega)$ that is basically the permittivity. This limit define the following dispersion relation,

$$
1-\sum_{j} \frac{\omega_{p j}^{2}}{k^{2}} \int d \vec{v} \frac{\vec{k} \bullet \nabla_{\bar{v}} f_{0 j}(\vec{v})}{\vec{k} \bullet \vec{v}-\omega}=0
$$


Where $\vec{k}$ is the wave number, $\omega_{p}$ is the plasma frequency, and the frequency $\omega$ can be complex.

To examining stability properties of a system, equation 9 must be solved for the system distribution function $f_{o}(\vec{v})$. Solutions for $\varphi$ are in the form of $\varphi=\widetilde{\varphi} e^{-i(\omega-\vec{k} \bullet \vec{v}) t}$. Complex values of $\omega$ indicate that wave growth or damping occurs, depending on the sign of Im $(\omega)$. Landau showed ${ }^{3}$ that waves are damped in plasma with a Maxwellian distribution function, thus explaining observed damping of waves in an electron tube.

\section{Dispersion Relation for E-Cooler Electron and Ion Bunches}

Equation 9 is valid for infinite homogenous plasma with no external electric or magnetic fields. To adapt equation 9 for our case of interest, it is best to use an equation that includes external magnetic fields that is particular suitable for systems with cylindrical geometry ${ }^{4}$, which is sometimes referred to as the Harris dispersion relation. Harris derived this equation to ease micro-stability analysis in magnetic mirror device ${ }^{5}$, whose diameter to length ratio is of order unity. Hershcovitch modified that equation and adapted it to cylindrically shaped plasma systems, ${ }^{6,7,8,9}$ (gated electron traps, and EBIS devices) that are characterized by large lengths and tiny diameters. In particular, EBIS bares an additional similarity to PRF RHIC E-Cool bunches with its different lengths of electron and ion columns. The most suitable dispersion relation ${ }^{9}$ for our system is

$$
1-\sum_{j=i, e} \frac{\omega_{p j}^{2}}{k^{2}} \int d \vec{v}\left\{i R^{\prime}\left[k_{\|} \frac{\partial}{\partial v_{\|}}+\left(\omega-k_{\|} v_{\|}\right) \times \frac{1}{v_{\perp}} \frac{\partial}{\partial v_{\perp}}-\frac{1}{\Omega_{j}}\left(k_{\theta} \frac{\partial}{\partial r}-k_{r} \frac{1}{r} \frac{\partial}{\partial \theta}\right)\right]+\frac{1}{v_{\perp}} \frac{\partial}{\partial v_{\perp}}\right\} f_{o j}\left(\vec{r}, v_{\perp}, v_{\|}\right)=0
$$

Where $R^{\prime}$ sometimes referred to as the propagator is

$$
R^{\prime}=\sum_{n=-\infty}^{\infty} \frac{i J_{n}^{2}\left(k_{\perp} v_{\perp} / \Omega_{j}\right)}{\omega-k_{\|} v_{\|}-n \Omega_{j}}
$$

Parallel and perpendicular is with respect to the magnetic field. Summation over species is over ions and electrons; $\mathrm{J}$ in equation 10 is a Bessel function (not current density).

Based on ion bunch and cooler parameters as well as assuming Gaussians for spatial radial distribution functions, ion and electron distribution functions are

$$
\begin{aligned}
& f_{o i}=\frac{m}{2 \pi \kappa T_{\perp}}\left(\frac{m}{2 \pi \kappa T_{\|}}\right)^{1 / 2} \exp \left[-\frac{m}{2 \kappa}\left(\frac{v_{\perp}^{2}}{T_{\perp}}+\frac{v_{\|}^{2}}{T_{\|}}\right)\right] \exp \left(-\frac{r^{2}}{R^{2}}\right) \\
& f_{o e}=\frac{m}{2 \pi \kappa T_{\perp}}\left(\frac{m}{2 \pi \kappa T_{\|}}\right)^{1 / 2} \exp \left[-\frac{m}{2 \kappa}\left(\frac{v_{\perp}^{2}}{T_{\perp}}+\frac{v_{\|}^{2}}{T_{\|}}\right)\right] \exp \left(-\frac{r^{2}}{R^{2}}\right)
\end{aligned}
$$


Where $\mathrm{R}$ is the bunch radius. Substituting equations 11 into equation 10 yields (after some manipulations and after dividing numerator and denominator of the velocity exponent of the electron distribution function by $T_{\perp}$ and of the ion distribution function by $T_{\|}$; and noting that $T_{\perp}>>T_{\|}$for electrons),

$$
\begin{aligned}
& 2 \frac{\omega_{p e}^{2}}{k^{2} v_{\text {the }}^{2}}\left(1+\frac{\omega-\omega_{* e}}{\omega-n \Omega_{e}}\right)\left\{1-\frac{\omega-n \Omega_{e}}{\omega}\left(1-\frac{T_{\| e}}{T_{\perp e}}\right)\right\} \sum_{n=-\infty}^{\infty} \exp \left(-b_{e}\right) I\left(b_{e}\right) Z\left(\frac{\omega-n \Omega_{e}}{k_{\|} v_{\text {the }}}\right)+ \\
& 2 \frac{\omega_{p i}^{2}}{k^{2} v_{t h i \|}^{2}}\left(1+\frac{\omega-\omega_{* i}}{\omega-n \Omega_{i}}\right)\left\{1-\frac{\omega-n \Omega_{i}}{\omega}\left(1-\frac{T_{\| i}}{T_{\perp i}}\right)\right\} \sum_{n=-\infty}^{\infty} \exp \left(-b_{i}\right) I_{n}\left(b_{i}\right) Z\left(\frac{\omega-n \Omega_{i}}{k_{\|} v_{t h i \|}}\right)=\varepsilon \\
& =0
\end{aligned}
$$

Where $b_{j}=0.5\left(k_{\perp} v_{t h j \perp} / \Omega_{j}\right)^{2}$ and $\omega_{* j}=-0.5\left(k_{\perp} v_{t h e j}^{2} / \Omega_{j} R\right)$ is the frequency associated with "drift" waves. It should be noted that the perpendicular wave number is in the azimuthal direction due to the cylindrical symmetry of the system. $Z$ is the well-known plasma dispersion function ${ }^{10}$. Z function properties, its tabulation and power series expansion can be found in reference 10 , while its asymptotic behavior ${ }^{11}$ and other approximations ${ }^{12}$ were derived later. The $\mathrm{Z}$ function is defined as,

$$
Z(\varsigma) \equiv \pi^{1 / 2} \int_{-\infty}^{+\infty} \frac{d t \exp \left(-t^{2}\right)}{t-\varsigma}=2 i \exp \left(-\varsigma^{2}\right) \int_{-\infty}^{i \varsigma} d t \exp \left(-t^{2}\right) \text {; Physically, } \varsigma=x+i y \text { is the ratio of }
$$

wave phase velocity to thermal velocity (obviously $\mathrm{x} \& \mathrm{y}$ are the real and imaginary arguments). In our case, $\varsigma>>1$, hence, the asymptotic series expansion of reference 11 can be used.

Although equation 12 is a dispersion relation in terms of known Bessel and plasma dispersion function, it cannot be solved analytically in its present form. In the next section, physical arguments are invoked to eliminate modes (and hence terms in the dispersion relation) that could not possibly occur.

\section{Mode Analysis}

Before proceeding with eliminating terms in equation 12 , justification is made for the exclusion of axial electric field effects. Electric fields can be incorporated by adding a term in the denominator of the propagator as done for internal fields ${ }^{13}$, externally induced fields ${ }^{7}$, or as it was very cleverly done ${ }^{14}$ by adding an electric field term to distribution functions that modifies self-consistently. Utilizing any of these methods to include the axial electric fields would significantly complicate an already complex problem. But the electric field was shown to have a minute effect ${ }^{15}$ on the ion parallel (or electron) energy spread. Therefore, it is simpler to use quasi-linear argument to claim that those electric fields have insignificant affects on particle orbits (regarding the propagator), and negligible effect on the distribution functions. Should the later become even slightly significant (note that a hurricane is a slight deviation from a Maxwellian), it raises the 
potential for beam instabilities. However, these instabilities have density threshold that well above our densities.

Next various frequencies (modes) are evaluated and compared to bunch parameters to determine their relevance. To be consistent with previous computations ${ }^{15}$, the following parameters are considered: ion and electron bunches that 30 meters and 3 meters long respectively, bunch radius for both ions and electrons is $2 \mathrm{~mm}$. For a $10 \mathrm{nC}$ electron bunch, the electron plasma density is $1.66 \times 10^{15} \mathrm{~m}^{-3}$, and the electron plasma frequency $\omega_{p e}=3.66 \times 10^{8} \mathrm{~Hz}$ for a cylindrically shaped bunch (the values are $2.2 \times 10^{15} \mathrm{~m}^{-3}$ and $\omega_{p e}=4.2 \times 10^{8} \mathrm{~Hz}$ for ellipsoid electron bunch). In either case the oscillation period is over $2 \mathrm{nsec}$, which longer than the $1 \mathrm{nsec}$ interaction time. For $10^{9}$ (ions per bunch) fully stripped gold ions, $\omega_{p i}=1.9 \times 10^{6} \mathrm{~Hz}$. In a Tesla magnetic field, the ion and electron cyclotron frequencies are $\Omega_{i}=6.1 \times 10^{6} \mathrm{~Hz}$ and $\Omega_{e}=2.8 \times 10^{10} \mathrm{~Hz}(28 \mathrm{GHz})$. Thus, only the electrons are magnetized (by plasma physics definition requiring $\Omega \gg$ frequencies like collisions or in this case $1 / \tau$ ).

Since ions are unmagnetized, $\omega_{* i}$ can be neglected. For electrons $\omega_{* e}=3.76 \times 10^{8}$ (for a transverse $1 \mathrm{KeV}$ electron temperature), therefore, all drift modes can be neglected (since ions are not even magnetized). Hence, no micro-instabilities driven by density gradients can occur, since the interaction time is shorter than oscillation period or growth rate of any of those instabilities.

Therefore, the last remaining source of free energy that could drive a micro-instability is velocity space anisotropy. If $T_{\|}>>T_{\perp}$ for both ions and electrons, equation 12 would yield only modes that are either oscillatory or damped, since $\operatorname{Im}(\omega)<0$ for all values. However, in our case $T_{\perp} \gg T_{\|}$for electrons.

With all these approximations, assuming that both ions and electrons have bi-Maxwellian energy distributions and noting that $T_{\perp} \gg T_{\|}$for electrons, equation 12 becomes,

$$
\begin{aligned}
& 0=1+2 \frac{\omega_{p e}^{2}}{k^{2} v_{\text {thel }}^{2}}\left(1+\frac{\omega}{\omega-n \Omega_{e}}\right)\left\{1-\frac{\omega-n \Omega_{e}}{\omega}\right\} \sum_{n=-\infty}^{\infty} \exp \left(-b_{e}\right) I\left(b_{e}\right) Z\left(\frac{\omega-n \Omega_{e}}{k_{\|} v_{t h e \|}}\right)+ \\
& 2 \frac{\omega_{p i}^{2}}{k^{2} v_{t h i \|}^{2}} \frac{T_{\| i}}{T_{\perp i}} \sum_{n=-\infty}^{\infty} \exp \left(-b_{i}\right) I_{n}\left(b_{i}\right) Z\left(\frac{\omega}{k_{\|} v_{t h i \|}}\right)
\end{aligned}
$$

Both transverse and longitudinal ion temperatures are less than $1 \mathrm{MeV}$ with expected velocities of less than $4 \times 10^{5} \mathrm{~m} / \mathrm{s}$, which is equivalent to a velocity of about $1 \mathrm{eV}$ electrons. Due to the geometry, $k_{\perp}>k_{\|}$(for ions $k_{\|} \rightarrow 0$ ) is expected. Therefore, ion Landau damping can be neglect. Next, angle $\theta$ is defined to be the angle that the $\mathrm{k}$ vector makes with the magnetic field. With these assumptions, equation 13 becomes, 


$$
\begin{aligned}
& 1+\omega_{p e}^{2} \frac{1-I_{0}(b) \exp (-b)}{k^{2} v_{\perp}^{2}}-\frac{\omega_{p e}^{2} \cos ^{2} \theta}{\omega^{2}} I_{0}(b) \exp (-b)-\omega_{p e}^{2} \cos ^{2} \theta \sum_{n \neq 0} \frac{I_{n}(b) \exp (-b)}{\left(\omega-n \Omega_{e}\right)^{2}}- \\
& \frac{\omega_{p e}^{2}}{k^{2} v_{\perp}^{2}} \sum_{n \neq 0} \frac{\omega I_{0}(b) \exp (-b)}{\omega-n \Omega_{e}}=0
\end{aligned}
$$

Terms in equation 14 are grouped in such a way that if the oscillation frequency is near a cyclotron harmonic, all terms in the summations other than that " $n$ " number can be neglected. Also, it is important to note that $b \cong\left(k_{\perp} \rho_{e}\right)^{2}$ where $\rho_{e}$ is the electron gyroradius. In our case largest $k_{\perp}=\frac{1}{r_{\text {plasma }}} \approx \frac{2}{2.4} \mathrm{~mm}^{-1}$. Since for $1 \mathrm{KeV}$ electrons $\rho_{e}=7.5 \times 10^{-2} \mathrm{~mm}$, $k_{\perp} \rho_{e}$ is usually a small number.

Equation 14 is next analyzed in the limit of small $k_{\perp} \rho_{e}$ for resonant and nonresonant instabilities. For the nonresonant case, $I_{n}(b) \exp (-b)$ is numerically small (observing that for $n \neq 0, I_{n}(b) \exp (-b)$ does not exceed 0.2 for all $\mathrm{n} \& \mathrm{~b}$ ), equation 14 reduces to

$\frac{\omega_{p e}^{2} \cos ^{2} \theta}{\omega^{2}} I_{0}(b) \exp (-b)=1+\omega_{p e}^{2} \frac{1-I_{0}(b) \exp (-b)}{k^{2} v_{\perp e}^{2}}$

Solution for equation 15 is

$$
\omega^{2}=k_{\|}^{2} \frac{T_{\perp e}}{m_{e}} \cdot \frac{I_{0}(b) \exp (-b)}{1-I_{0}(b) \exp (-b)+k^{2} \frac{v_{\perp e}^{2}}{\omega_{p e}^{2}}}
$$

For complex roots to occur, the denominator of equation 16 must be negative. But, since for our values $I_{n}(b) \exp (-b) \geq 1$, the term $k^{2} \lambda_{D}^{2}$ needs to be miniscule (note that $\left.v_{\perp e}^{2} / \omega_{p e}^{2} \approx \lambda_{D}^{2}\right)$. But since $\lambda_{D}$ is close to the bunch diameter, very long wavelengths are required to satisfy instability condition. This cannot happen due to the small radial dimension of the bunches.

Now consider the $\omega \approx n \Omega_{e}$ case in equation 14 . Choosing only one $\mathrm{n}$ number, solution is straightforward,

$\omega \approx n \Omega_{e}$, and the growth rate $\gamma=\frac{\sqrt{3}}{2}\left(\frac{1}{2} \bullet \frac{I_{n}}{I_{0}}\right)^{2 / 3} \Omega_{e}$. These parameters are of time scales that can affect the RHIC E-Cooler. However, these values must be substituted into equation 12 to first compute the necessary values of the plasma density and then calculate the temperature anisotropy needed for instability (drift and rotation frequencies can still 
be neglected; slow compared to the interaction). Doing yields necessary conditions for instability to be

$$
\frac{\omega_{p e}^{2}}{\Omega_{e}^{2}} \geq 1
$$

And

$$
\frac{T_{\| e}}{T_{\perp e}}<\left(k_{\perp} \rho_{e}\right)^{2 / 3}
$$

Conditions set by equation 18, can be met in the RHIC E-Cooler. However, equation 17 has a required critical density (that can trigger this instability), which is a factor of over 70 above the electron density in the RHIC E-Cooler.

In the literature, instabilities due to temperature anisotropy ${ }^{5,16,17,18}$ were examined due to concern of their occurrence in mirror devices as a consequence of their inherent velocity space loss cone. Even though these instabilities were analyzed using kinetic theory, cases where the wave phase velocity was much larger than thermal velocity were referred to as "hydrodynamic instabilities."

\section{Discussion}

In conclusion, RHIC E-Cooler is stable against the only microinstability that can develop in one interaction period. Within the limits of plasma kinetic theory, confidence in the obtained results should be fairly high since they are consistent with other results that can be found in the literature regarding instabilities due to temperature anisotropy ${ }^{5,16,17,18}$. In these papers, $\gamma \approx \Omega_{e}$ (occurring at $k=\rho_{e}^{-1}$ ), and conditions for stability are $\frac{T_{\|}}{T_{\perp}}<\frac{1}{2}$, or a condition linking $\beta^{*}$, the ratio of plasma to magnetic field pressure, to the temperature anisotropy to be $\frac{T_{\perp \perp}}{T_{\|}}-1>\frac{1}{2 \beta^{*}}$. Given the geometrical differences (analyzed mirror devices have comparable lengths and diameters), the results obtained in this note are plausible.

Past analysis ${ }^{19}$ of instabilities due to ion-electron oscillations cannot occur, since the calculation involves some frequencies that are too low to have an effect during the $1 \mathrm{nsec}$ interaction time. Similarly, instabilities due to secondary particles should not be of concern during the $1 \mathrm{nsec}$ interaction time (Budker-Buneman and beam plasmas have frequencies that are a small fraction of the plasma frequency).

In the future, it may be worthwhile to perform a similar MHD analysis, and to consider the effect of ballistic modes. The latter may occur due to high excitation rate (repeated interaction between electron and ion bunches), echoes might have the potential to cause some problems, even though unstable echoes were never observed or even predicted to occur. However phase mixing between echoes [arising from ballistic modes] and other 
perturbed elements in the distribution function might lead to instabilities. But, this case is radically different from anything that was previously looked at; a completely new theory must be developed.

Finally some general comments regarding validity and experimental verification of pertinent theoretical analysis of various instabilities. Stability has been one of the most controversial topics in plasma physics research. Consequently, no results should be viewed as certain. Part of the reason for skepticism stems from the experience of the U.S. fusion community had with regard to stability of stellarators. During the 1950's, an anomalously high rate of particle loss (referred to as particle pumpouts) was observed in the Princeton stellarators. This phenomenon became a catalyst for major developments in plasma kinetic theory, since plasma microinstabilities were believed to be the reason for the pumpouts. In the 1970's, it was discovered that the reason for the pumpouts was poor magnetic field quality of the early stellarators, which caused particles to drift out. With greatly improved computer codes, a new generation of stellarators was built in the 1970's that matched the performance of comparable tokamaks. Much worst was the experience of the EBIS community ${ }^{20}$, where poor performance had being blamed on plasma instabilities rather than seeking simpler explanations like misalignment.

Nevertheless, there were also great achievements like those in the subfield of plasma physics that dealt with stability of magnetic mirror devices. Both MHD and kinetic theories successfully explained major macro and microinstabilities, for which remedies were eventually found. Present analysis is similar to what was done in mirror devices.

Much of the prior work on stability analysis of electron beam coolers involved space charge ion-electron oscillations, or relative oscillations of ion and electron columns. Those problems were treated mathematically as classical anharmonic oscillators without using two time scale approximations. Although correct results might have still been generated, the computations have a potential flaw. In a book by Osrzag and Bender (based on works by Kolmogoroff, Cole, as well as Bogoliubov \& Mitroposky) on this topic, it is shown that time secularities can appear due to a mathematical art-effect in perturbation theory, where a lower order term can drive a higher order equation at the resonant frequency. To ensure that this does not occur, a two time scale analysis must be performed. Additionally, poor correlation ${ }^{21}$ was reported to exist between stability analysis and experimental results.

\section{Conclusion}

1. Within the limits of plasma kinetic theory, the only microinstability that can potentially occur is one due to anisotropy of the electron temperature in a magnetic field. However, the instability has a density threshold that electron density is a factor of over 70 larger than that of the RHIC E-Cooler.

2. A comparable macroscopic theory analysis, and/or a novel theory that includes plasma echoes may be worth pursuing.

3. It might be worthwhile to revisit prior work. 


\section{Acknowledgement}

The author is thankful to Ilan Ben-Zvi for suggesting this problem.

\section{References}

1. Computed from values provided by Ilan Ben-Zvi private communication 2003.

2. A. A. Vlasov, J. Phys. (USSR) 9, 25 (1945).

3. L. D. Landau, J. Phys. (USSR) 10, 25 (1946).

4. E. G. Harris, J. Nucl. Energy, Part C 2, 138 (1961).

5. E. G. Harris, Phys. Rev. Letters, 2, 34 (1959).

6. A. Hershcovitch and P. A. Politzer, Phys. Rev. Letters, 36, 1365 (1976).

7. A. Hershcovitch and P. A. Politzer, Phys. Fluids, 22, 249 (1979).

8. A. Hershcovitch, Physica Scripta T71, 34 (1996).

9. A. Hershcovitch, Rev. Sci. Instrum. 69, 668 (1998).

10. B. D. Fried and S. C. Conte, The Plasma Dispersion Function, Academic Press New York (1961).

11. R.W. Landau and S. Cuperman, J. Plasma Physics 6, 495 (1971).

12. B. D. Fried, C. L. Hedrick, and J. McCune, Phys. Fluids 11, 249 (1968).

13. C. T. Dum and T. H. Dupree, Phys. Fluids 13, 2064 (1970).

14. A. B. Mikhailovskii, Theory of Plasma Instabilities Vol. I, Consultant Bureau, New York (1974).

15. A. Hershcovitch, Parallel Ion Energy Spread Due to Interaction with an Electron Bunch in the RHIC Electron Beam Cooling Solenoid, CA/AP Note \# 102, June 2003.

16. E. S. Weibel, Phys. Rev. Lett. 2, 83 (1959).

17. H. P. Furth, Phys. Fluids 6, 48 (1963)

18. M. N. Rosenbluth, Microinstabilities Plasma Physics, IAEA, Vienna (1965).

19. V. Parkhomchuk and I. Ben-Zvi, C-A/AP note \# 47 April 2001.

20. See for example panel discussion, N. Rostoker, moderator, in "Proceedings of International Symposium on Electron Beam Ion Sources and Their Applications", A. Hershcovitch, Editor, American Institute of Physics Conference Proceedings No. 188, American Institute of Physics, New York, 1989, pp. 197 - 187.

21. J. Bosser, in a talk given at the $7^{\text {th }}$ EBIS symposium in Gelnhausen, Germany and private 1996. 